\title{
Secure Data Transfer through DNA Cryptography using Symmetric Algorithm
}

\author{
Bonny B. Raj \\ Research Scholar \\ Department of Computer \\ Science and Engineering \\ Hindustan University \\ Chennai, India
}

\author{
J. Frank Vijay, PhD \\ HoD in Information Technology \\ KCG College of Engineering \\ and Technology \\ Chennai, India
}

\author{
T. Mahalakshmi, PhD \\ Principal \\ Sree Narayana Institute of \\ Technology, Vadakkevila, \\ Kollam, India
}

\begin{abstract}
This paper presents a novel symmetric algorithm in the area of DNA cryptography. Secure Data Transfer is an important factor for data transmission. The transmission of information can be of local or of global scope. But it is mandatory to secure information from unauthorized access. Security is very important factor encryption. This method proposes a secured symmetric key generation process which generates initial cipher and this initial cipher is then converted into final cipher using random key generated DNA sequences, so as to make it complicated.
\end{abstract}

\section{General Terms}

Bio- inspired computing, Security, Algorithms et. al.

\section{Keywords}

Bio-inspired computing, Symmetric Encryption, Random key, Symmetric key.

\section{INTRODUCTION}

Security of valuable information is ensured using cryptographic techniques. Using such techniques enables the sender for secure transmission as well as storage of sensitive information through the internet. A cryptographic system applies encryption of information and thus produces an encrypted output which may be meaningless to an intruder who has no knowledge of the key. The knowledge of the key is essential factor for encryption and decryption process. The fundamental tool for cryptography is a simple function which is one-way and is easy to compute but very hard to get invert [1].

Encryption and decryption phases of cryptography are determined by keys. Based on keys, cryptographic systems can be classified as Symmetric Key Cryptography (SKC) and Asymmetric Key Cryptography (AKC) also known as public key cryptography [2]. Symmetric Key Cryptographic system uses single key for both encryption and decryption. In Asymmetric Key Cryptographic system different keys are used for both encryption and decryption.

SKC is based on sharing secrecy of the key, where as AKC is based on personal secrecy of the key [3].Generally in the former symbols are permuted or substituted where as in the later numbers are manipulated using functions. In the SKC key is shared between the sender and the receiver. In AKC the key is personal and is known to themselves. The difficulty in SKC approach is the distribution of the key where as in AKC there is no such distribution of the key [12].
DNA Cryptography is generally defined as hiding data in DNA sequence. The advantages of this is as follows [9].

a) By supporting large parallelism which helps to improve computational speed.

b) The molecules of DNA acts as carrier of transmission with large capacity.

c) Power consumption is small.

In the present work a novel SKC algorithm using DNA cryptography is proposed which does not need any DNA Chromosome OTP structure as seen in paper [12].

The remaining part of this paper is organized as follows: Section 2 gives background information relevant to the proposed work which is followed by literature review in section 3.In section 4 a proposed algorithm is elucidated followed by discussion in section 5 and conclusion in section 6.

\section{BACKGROUND}

This section describes some terms which are frequently used in this paper.

\subsection{DNA (De-oxyribo Nucleic acid)}

DNA refers to De-oxyribo nucleic acid is a nucleic acid which contains genetic information. It is used for the growth as well as functioning of all living organisms. They have two long chains of nucleotide which has a double helical structure [5].It a collection of most complex organic molecules. The instructions in DNA is required for the construct other components of cells such as protein, RNA molecules etc [16] The segments in DNA that hold genetic information are known as genes. These sequences are used modifying the use of this genetic information, just as a string of binary information which is encoded with zeroes and ones. DNA strand encoding include four bases which are represented by letters A (Adenine), $\mathrm{T}$ (Thymine), $\mathrm{C}$ (Cytosine) and $\mathrm{G}$ (Guanine) [15].Each alphabet is related to a nucleotide. They are very long. For instance, the DNA sequence of length 10 nucleotides long can be represented as ATCGAATTCG.

\subsection{DNA Cryptography}

DNA cryptography is a new promising direction in cryptography research that emerged with the evolution of DNA computing. DNA can be used for storing, transmitting and computation of information [6]. Parallelism and extraordinary information density inbuilt in this molecule are exploited for encryption and decryption purpose[14]. Different DNA based algorithm are available for this purpose. In this paper, the research conducted by various authors related to this discipline is taken into consideration. This shows, how DNA cryptography uses DNA as a 
computational tool to manipulate for encryption and decryption[7]. Research work can be done on DNA by two ways either by molecule or by simulation [8].

\subsection{Symmetric key Algorithm}

Symmetric or Secret key can be classified as stream ciphers or block ciphers. Stream ciphers operate on a single bit (byte or computer word) at a time, and implement some form of feedback mechanism so that the key is constantly changing [R1].A block cipher encrypts a block of data at a time using the same key on each block. There are a number of other secret-key cryptography algorithms that are also in use today like CAST-128 (block cipher), RC2 (block cipher) RC4 (stream cipher), RC5 (block cipher), Blowfish (block cipher), Two fish (block cipher). In 1997, NIST initiated a process to develop a new secure cryptosystem for U.S. government applications. The proposed system use symmetric key stream cipher algorithm.

\section{LITERATURE REVIEW}

The literature study revealed the existence of the paper titled" "Yet Another Encryption Algorithm" (YAEA) developed by Saeb and Baith [13] which is based on a conventional symmetric key algorithm. The study also reveal the existence of another paper based on DNA computing in the field of cryptography[12].

It was found that the field of DNA Cryptography belongs to the area of Bio-inspired computing methodologies. Biologically-inspired computing (BIS) is the study which deals with the topics of connection, social behavior and its emergence [4]. Bio-inspired computing methodologies are designed to solve specific problem, biological system, which follows a specific procedure and can has similar properties. Some forms shows complex behavior and rules. Complexity increases until the result is something complex. Final results are often completely different from what the original rules would be expected to produce.

The first DNA based cryptographic technology was seen in [6].Somewhat similar to the proposed work is seen in [13].

Cryptosystems which use a secret random OTP are known to be perfectly secure [12]. OTP encryption uses a large nonrepeating set of truly random key letters. Each pad is used exactly once, for only one message.

The survey also revealed the different DNA data encryption algorithm which uses traditional mathematical operations and/or data manipulating DNA techniques [10].The proposed method is also an application in this area.

\section{PROPOSED SYSTEM}

Proposed system is a new encryption algorithm based on random key generation of DNA pattern. There are three stages in this algorithm- Encryption, Random Key Generation and Decryption.

In the first stage the source data is encrypted which is the input to the second stage. In second stage random key is generated, say $\mathrm{Pk}$ which is used for next level of encryption. In the third stage decryption process takes place.

Fig: 1 gives the flow chart of the stages I and II of the proposed system and the Fig: 2 gives the flow chart of the stage III of the proposed system.

The remaining part of this section explains in details each of these three stages.

\subsection{Encryption}

This is the stage one of the proposed algorithm. The input for this is the source data which is in the text format. Each character of the source data is converted to its corresponding ASCII value which is in turn converted to its binary value in Fig 1 first three blocks corresponds to this stage. This process is explained in details as follows.

Consider the message the $\mathrm{M}$ to be transmitted to the receiver, the different steps for encryption is as follows

Step 1: The text to be transmitted is first converted to ASCII code (in decimal format).

Step 2: These decimal values are grouped into blocks.

Step 3: This encoded ASCII message (decimal values) is then converted to binary format ( 0 's and 1 's).

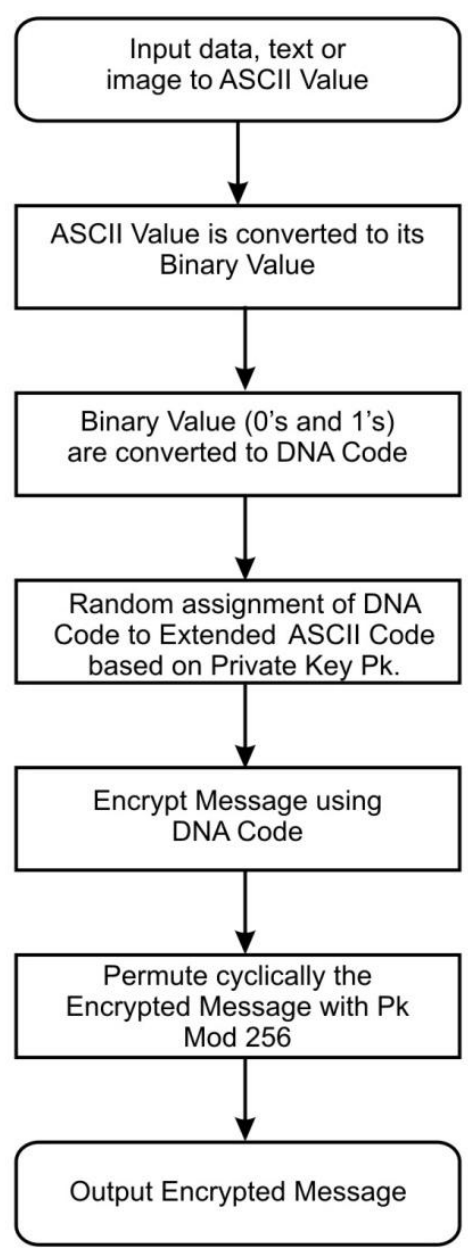

Fig1: Stage I and II of the Proposed System.

\subsection{Random Key Generation}

This is the second stage in the proposed system. In this stage a random key say $\mathrm{Pk}$ in the range 1 to 256 is generated for further encryption. Corresponding to each value of $\mathrm{Pk}$ an index table is of size 256 is generated where each value of the table corresponds to a combination of A, T, G and C.When $\mathrm{Pk}=1$ the index table generated is as shown in Table 1 . The size of the index table is 256 which corresponds to the permutation of four characters. A, T, G and C. As the value of $\mathrm{Pk}$ varies the index table will also varies. 
The encrypted binary data from the stage 1 is the input for this stage. First the input data is selected as pairs. Each pairs is replaced by the DNA nucleotides A, T, G, and C corresponding to the values $00,01,10$ and 11 .

From the index table the index key corresponding to the combination of A, T, G, and C is obtained which is the final encrypted value corresponding to the character of source data.

In Fig:1 blocks 4,5,6,7 represents this stage. The value of $\mathrm{Pk}$ is also transmitted along with encrypted character from this source data. Given below in detail the various steps involved in this stage.

Step 4: Encrypted binary data obtained from the first stage is considered in the form of pairs. Since the encrypted data is binary these pairs will be either 00 or 01 or 10 or 11.These pairs are substituted by $\mathrm{A}$ for $00, \mathrm{~T}$ for $01, \mathrm{G}$ for 10 , and $\mathrm{C}$ for 11.

Step 5: Generate Pk

Step 6: Corresponding to the value of Pk generate index table.

Step 7: The A, T, G and C obtained from step 4 is used to find the final encrypted key from the index table generated in step 6.

Table 1: Random Generated Key

\begin{tabular}{|c|c|c|c|c|c|c|c|c|c|c|c|c|c|c|c|}
\hline 1 & AAAA & 33 & CAAA & 65 & GAAA & 97 & TAAA & 129 & AGAA & 161 & CGAA & 193 & GGAA & 225 & TGAA \\
\hline 2 & AAAC & 34 & CAAC & 66 & GAAC & 98 & TAAC & 130 & AGAC & 162 & CGAC & 194 & GGAC & 226 & TGAC \\
\hline 3 & AAAG & 35 & CAAG & 67 & GAAG & 99 & TAAG & 131 & AGAG & 163 & CGAG & 195 & GGAG & 227 & TGAG \\
\hline 4 & AAAT & 36 & CAAT & 68 & GAAT & 100 & TAAT & 132 & AGAT & 164 & CGAT & 196 & GGAT & 228 & TGAT \\
\hline 5 & AACA & 37 & CACA & 69 & GACA & 101 & TACA & 133 & AGCA & 165 & CGCA & 197 & GGCA & 229 & TGCA \\
\hline 6 & $A A C C$ & 38 & $C A C C$ & 70 & $\mathrm{GACC}$ & 102 & TACC & 134 & AGCC & 166 & $\mathrm{CGCC}$ & 198 & GGCC & 230 & TGCC \\
\hline 7 & AACG & 39 & $C A C G$ & 71 & GACG & 103 & TACG & 135 & AGCG & 167 & CGCG & 199 & GGCG & 231 & TGCG \\
\hline 8 & AACT & 40 & CACT & 72 & GACT & 104 & TACT & 136 & AGCT & 168 & CGCT & 200 & GGCT & 232 & TGCT \\
\hline 9 & AAGA & 41 & CAGA & 73 & GAGA & 105 & TAGA & 137 & AGGA & 169 & CGGA & 201 & GGGA & 233 & TGGA \\
\hline 10 & AAGC & 42 & CAGC & 74 & GAGC & 106 & TAGC & 138 & AGGC & 170 & CGGC & 202 & GGGC & 234 & TGGC \\
\hline 11 & AAGG & 43 & CAGG & 75 & GAGG & 107 & TAGG & 139 & AGGG & 171 & CGGG & 203 & GGGG & 235 & TGGG \\
\hline 12 & AAGT & 44 & CAGT & 76 & GAGT & 108 & TAGT & 140 & AGGT & 172 & CGGT & 204 & GGGT & 236 & TGGT \\
\hline 13 & AATA & 45 & CATA & 77 & GATA & 109 & TATA & 141 & AGTA & 173 & CGTA & 205 & GGTA & 237 & TGTA \\
\hline 14 & AATC & 46 & CATC & 78 & GATC & 110 & TATC & 142 & AGTC & 174 & CGTC & 206 & GGTC & 238 & TGTC \\
\hline 15 & AATG & 47 & CATG & 79 & GATG & 111 & TATG & 143 & AGTG & 175 & CGTG & 207 & GGTG & 239 & TGTG \\
\hline 16 & AATT & 48 & CATT & 80 & GATT & 112 & TATT & 144 & AGTT & 176 & CGTT & 208 & GGTT & 240 & TGTT \\
\hline 17 & ACAA & 49 & CCAA & 81 & GCAA & 113 & TCAA & 145 & ATAA & 177 & CTAA & 209 & GTAA & 241 & TTAA \\
\hline 18 & $A C A C$ & 50 & CCAC & 82 & $\mathrm{GCAC}$ & 114 & TCAC & 146 & ATAC & 178 & CTAC & 210 & GTAC & 242 & TTAC \\
\hline 19 & ACAG & 51 & CCAG & 83 & GCAG & 115 & TCAG & 147 & ATAG & 179 & CTAG & 211 & GTAG & 243 & TTAG \\
\hline 20 & ACAT & 52 & CCAT & 84 & GCAT & 116 & TCAT & 148 & ATAT & 180 & CTAT & 212 & GTAT & 244 & TTAT \\
\hline 21 & $\mathrm{ACCA}$ & 53 & $\mathrm{CCCA}$ & 85 & GCCA & 117 & TCCA & 149 & ATCA & 181 & CTCA. & 213 & GTCA & 245 & TTCA \\
\hline 22 & $\mathrm{ACCC}$ & 54 & $\mathrm{CCCC}$ & 86 & $\mathrm{GCCC}$ & 118 & $\mathrm{TCCC}$ & 150 & ATCC & 182 & СТCC & 214 & GTCC & 246 & TाCC \\
\hline 23 & $A C C G$ & 55 & $\mathrm{CCCG}$ & 87 & GCCG & 119 & TCCG & 151 & ATCG & 183 & CTCG & 215 & GTCG & 247 & TTCG \\
\hline 24 & ACCT & 56 & CCCT & 88 & GCCT & 120 & TCCT & 152 & ATCT & 184 & СТCT & 216 & GTCT & 248 & TातT \\
\hline 25 & $\mathrm{ACGA}$ & 57 & CCGA & 89 & GCGA & 121 & TCGA & 153 & ATGA & 185 & CTGA & 217 & GTGA & 249 & TTGA \\
\hline 26 & $\mathrm{ACGC}$ & 58 & CCGC & 90 & GCGC & 122 & TCGC & 154 & ATGC & 186 & CTGC & 218 & GTGC & 250 & TTGC \\
\hline 27 & ACGG & 59 & CCGG & 91 & GCGG & 123 & TCGG & 155 & ATGG & 187 & CTGG & 219 & GTGG & 251 & TTGG \\
\hline 28 & ACGT & 60 & CCGT & 92 & GCGT & 124 & TCGT & 156 & ATGT & 188 & CTGT & 220 & GTGT & 252 & TTGT \\
\hline 29 & ACTA & 61 & CCTA & 93 & GCTA & 125 & TCTA & 157 & ATTA & 189 & CTTA & 221 & GTTA & 253 & ताTA \\
\hline 30 & ACTC & 62 & ССТC & 94 & GCTC & 126 & ТСТС & 158 & ATTC & 190 & CTTC & 222 & GTTC & 254 & ताTC \\
\hline 31 & ACTG & 63 & CCTG & 95 & GCTG & 127 & TCTG & 159 & ATTG & 191 & CTTG & 223 & GTTG & 255 & TITG \\
\hline 32 & $\mathrm{ACT}$ & 64 & $\mathrm{CCTT}$ & 96 & GCTT & 128 & TCTT & 160 & ATTा & 192 & CTIT & 224 & GTTा & 256 & TTTT \\
\hline
\end{tabular}

\subsection{Decryption}

Decryption is a process of converting encrypted text back to original text. Only an authorized user can decrypt data because decryption requires a secret key or password.

The first block of decryption receives the encrypted message. The second block generate $\mathrm{Pk}$ value. The third block receives the encrypted DNA code and converts to its corresponding binary values. These pairs are substituted by 00 for A, 01 for $\mathrm{T}, 10$ for $\mathrm{G}$, and 11 for $\mathrm{C}$. The fourth block the arrange binary values to block. The fifth block convert binary value to its Extended ASCII Value and the sixth block convert Extended ASCII value back to the original data or the decrypted message.
Step 1: The DNA sequences are separated from primers (start primer and end primer), since the message is in between them.

Step 2: The DNA nucleotides A, T, G, and C characters are substituted accordingly $(00,01,11,10$ respectively).

Step 3: Then they are converted into ASCII code and then the message is received at receiver end. 


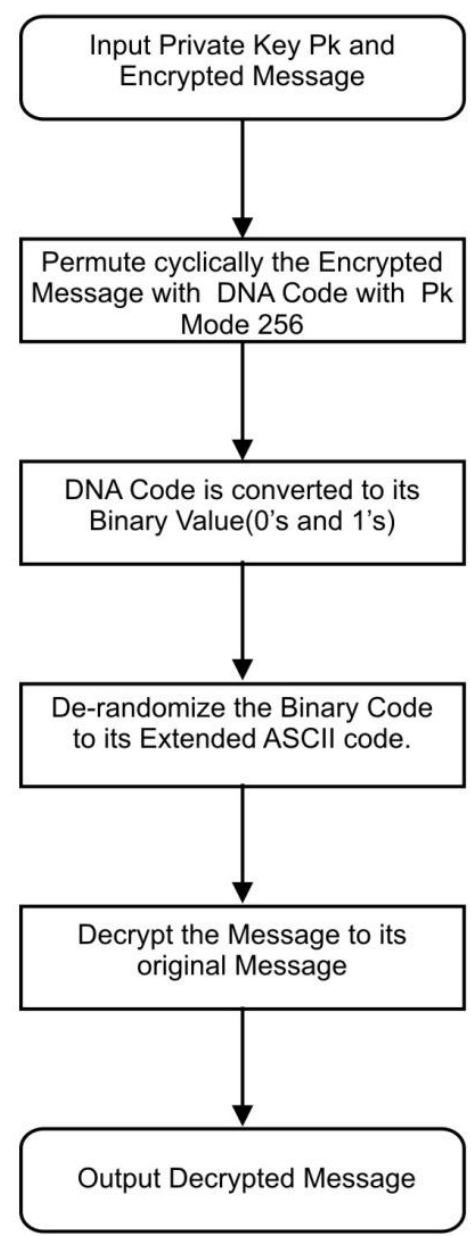

Fig 2: Decryption (Stage III of the Proposed system)

\subsection{Example}

The proposed system is explained with an example. Consider the source data be BOY

\subsubsection{Encryption Process}

Step 1: ASCII value of $\mathrm{B}=66, \mathrm{O}=79, \mathrm{Y}=89$

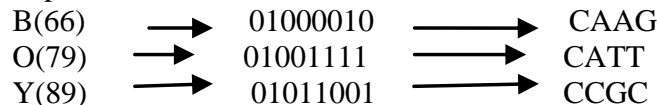

$\mathrm{Y}(89)$

0101100

CCGC

Step 2: After DNA Combination substitution the message BOY will become CAAG-CATT-CCGC.

Step 3: From random key substitution table the DNA combination will become $35, \mathbf{4 8}, \mathbf{5 8}$.

Step 4: So the final encrypted message or cipher text is 35,48,58.

\subsection{Decryption Process}

Step 1: Cipher text is $35,48,58$.

Step 2: Substitute random generated key at an instance

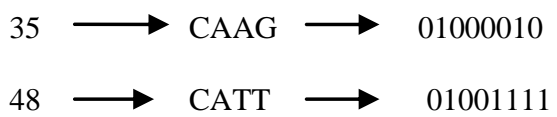

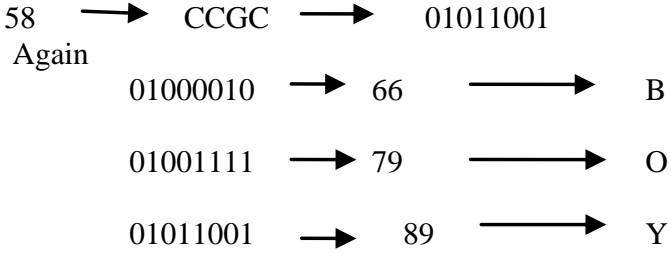

\section{DISCUSSION}

The proposed system is implemented using java as programming language and Window 7 as platform. Proposed system belongs to the modern symmetric key encryption system. Here each character is converted to an expanded set of characters. The specialty of the proposed system is that there is no need to use DNA chromosome or any other similar data during transmission, instead along with each character an inbuilt private key in the range of 1 to 256 will be transmitted.

The proposed system corresponds to the single character, two values are to be transmitted - one the encrypted integer value of $\mathrm{Pk}$ and the encrypted message. Based on the above discussion, one can conclude that security and the performance of the proposed algorithm are satisfactory for multi-level security applications of today's networks.

\section{CONCLUSION}

The proposed method of encoding is far better and faster than conventional cryptography like DES and other DNA based encryption algorithms. The proposal can be further enhanced to include in security mechanism of wireless networks and analyzing its performance to basic cryptanalytic attacks and comparing it with existing cryptosystems to know exactly how much improvement is achieved.

Security plays an important role in communication world. In order to protect the data from intruders, one need better security measures. The theoretical analysis shows that this method is more powerful against certain attacks. This method ensures confidentiality and data integrity over data transmission.

\section{REFERENCES}

[1] Adleman L M, Molecular Computation of Solutions to Combinatorial Problems, Science, Vol 266, pp 10211024, November 1994.

[2] K. Sireesha and V. Srujana, An overview and Analysis of Private \& Public Key, International Journal of Technological Exploration \& Learning, pp 281- 283, December 2013.

[3] Ayushi, A Symmetric Key Cryptographic Algorithm, International Journal of Computer Applications Vol 1, 2010.

[4] Bio-inspired computing: constituents and challenges, International Journal of Bio-Inspired Computation, Vol 1,pp 135-150, March 2009.

[5] Bonny B Raj and Panchami V, DNA Based Cryptography Using Permutation and Random Key Generation Method, International Journal of Innovative Research in Science, Engineering and Technology, Vol 3, pp 263-267, July 2014

[6] Gehani, T. LaBean, and J. Reif, DNA-Based Cryptography, Lecture Notes in Computer Science, Springer, Vol 2950, pp 167-188 2004.

[7] Rupali Soni and Gopal Prajapati, A Modern Review on Cryptographic Techniques, International Journal of 
Advanced Research in Computer Science and Software Engineering Vol 3, Issue 7, pp162-167 July 2013.

[8] http://www.blc.arizona.edu/molecular_graphics/dna_str ucture/dna_tutorial.html

[9] Shipra Jain and Dr. Vishal Bhatnagar, Analogy of Various DNA Based Security Algorithms Using Cryptography and Steganography, International Conference on Issues and Challenges in Intelligent Computing Techniques pp 285-291, 2014.

[10] Anu priya Agarwal, Praveen Kanth, Secure Data Transmission using DNA Encryption, Computer Engineering and Intelligent Systems Vol 5, pp 51-59, 2014.

[11] "Introduction to Public-Key Cryptography", an article availabledeveloper.netscape.com/docs/manuals/security/ pkin/contents.html.

[12] Olga Tornea, Monica Borda, Tatiana Hodorogea, and Mircea-Florin Vaida, Encryption System With Indexing
Dna Chromosomes Cryptographic Algorithm, Proceedings of the 7th IASTED International Conference, pp12-1, February 2010.

[13] Sherif T. Amin , Magdy Saeb and Salah El-Gindi, A DNA-based Implementation of YAEA Encryption Algorithm.

[14] S. T. Amin, M. Saeb, S. El-Gindi, A DNA-base Implementation of YAEA Encryption Algorithm, IASTEDInternational Conference on Computational Intelligence,San Francisco, pp 120-125, 2006.

[15] Tatiana Hodorogea, Mircea-Florin Vaida, Alternate Cryptography Techniques, ICCC05, Miskolc-Lillafured, Hungary, Vol. 1, pp 513-518, 2005.

[16] R. K. Wilson, The sequence of Homo sapiens FOSMID clone ABC14-50190700J6, submitted to http://www.ncbi.nlm.nih.gov, 2009. 